Research Article

\title{
Automatic Recognition and Correction of Volleyball Players' Release Angle Based on Feature Statistics
}

\author{
Rui Fang $\mathbb{D}$ \\ School of Physical Education, Xinyang Vocational and Technical College, Xinyang, Henan 464000, China \\ Correspondence should be addressed to Rui Fang; fangrui0213@xyvtc.edu.cn
}

Received 10 September 2021; Revised 11 October 2021; Accepted 26 October 2021; Published 27 November 2021

Academic Editor: Mu Zhou

Copyright (c) 2021 Rui Fang. This is an open access article distributed under the Creative Commons Attribution License, which permits unrestricted use, distribution, and reproduction in any medium, provided the original work is properly cited.

\begin{abstract}
The constant reform of the competition rules has promoted the innovation of volleyball techniques and tactics. In order to improve the training efficiency and competitive level of volleyball players, this study designed a volleyball player shooting angle automatic recognition and correction method based on the process of feature statistics. Firstly, the basic structure of the information acquisition system is analyzed, and the acquisition process is determined. Then, grayscale and binarization operations are carried out for color-moving images to separate their foreground and background, and a median filtering algorithm is used to remove the image noise. Then, the image pyramid of different sizes is generated by the filter. Based on setting the datum direction, the feature of volleyball shooting is extracted by using the line formula. On this basis, we construct a support vector machine (SVM) classifier to statistically classify the features, use the histogram additive kernel support vector machine method to obtain the lens angle recognition results, and correct the lens angle through feature point matching. Simulation experiments show that this method can effectively remove image noise and make the image signalto-noise ratio higher, and it can effectively identify whether volleyball players' release Angle is correct, to achieve the purpose of timely correction.
\end{abstract}

\section{Introduction}

Volleyball is a sport that makes the Chinese people proud, especially the Chinese women's national volleyball team, which has achieved impressive results in the past and has become a spirit of the times, inspiring the Chinese people to strive for self-improvement [1]. As the rules of the volleyball game system continue to change, it imposes higher demands on the physical quality and skill level of the players. Most of the traditional training methods rely on the coaches' observation and analysis of the angles of the players' shots through their own experience to find out the deficiencies and give training suggestions and instructions. The training in this way relies more on the coaches' own quality and is somewhat subjective.

In recent years, for various reasons, the development of volleyball in China has slowed down, which has caused widespread concern among enthusiasts. Modern sports involve high technology from players' equipment to training methods [2]. Therefore, the development of technology in volleyball training has become the goal of future development. Moreover, having information related to the angle of the athlete's shot is the first step to improve the technique [3].

In this study, the image acquisition system is used to collect the information of the shot angle of players, and the feature statistics are input into the Support Vector Machine (SVM) classifier to complete the shot angle recognition, and then the feature matching is performed on the collected information combined with the sample inventory in the information acquisition system. If it matches with the sample information, the shot angle meets the requirements; if not, it is corrected.

The reason for the use of feature statistics is that, in the use of mathematical statistics to study overall features, the focus is not on individuals in a population, but on the distribution of related features among different individuals in a population. 
This paper adopts this method to realize angle recognition, which reduces the complexity of recognition process and improves the accuracy of recognition.

\section{Information Acquisition and Processing of Shot Angle}

2.1. Information Acquisition System Structure and Acquisition Process. The motion information acquisition system, also known as the motion capture system, can realize the measurement, tracking, and recording of the target, which mainly includes the results of master controller, RF transceiver unit, network communication unit, and serial communication unit. The structure of shooting angle information collection system is shown in Figure 1.

2.1.1. Master Controller. The core part of the master controller is the master chip, and the selected microprocessor varies for different application scenarios. The microprocessor required in this study needs not to be portable and handheld. Therefore, in order to save resources, this study selects the embedded microcontroller as the system processor. In addition, the reader and the RFID both need to feature low-power consumption, so this study uses the ultra-low-power sixteen-bit industrial-grade microcontroller introduced by TI as the master chip.

2.1.2. RF Transceiver Unit. Generally, the acquisition system utilizes the acquisition method based on the signal reception strength, so the signal acquisition capability of the RF transceiver chip should be strong $[4,5]$. Based on a comprehensive analysis of the system requirements and functions, the $2.4 \mathrm{GHz}$ CC2500 is selected as the RF transceiver chip in this study because of its advantages of small size, low power consumption, and user-friendly design.

2.1.3. Network Communication Unit. This unit mainly refers to the Ethernet communication part, and the ENC28J60 from Microchip is selected in this study because it has only twenty-eight pins and has the advantages of low computational complexity and low cost [6].

2.1.4. Serial Communication Unit. The serial communication unit in this system uses MAX232, a level logic conversion chip to interconnect the UART port of the master controller with the PC to achieve communication.

Combining the structure of the above-mentioned information collection system, the construction of a volleyball information collection program is shown in Figure 2.

2.2. Image Information Preprocessing. The acquired volleyball motion images are preprocessed. Usually, the information contained in the image is complex, and the useful information generally accounts for only a small portion. In addition, there is also a certain amount of noisy information in the image due to the effect of illumination. The purpose of preprocessing is to improve the image quality and thus fundamentally improve the recognition effect. Image preprocessing in this study mainly includes grayscale, binarization, and filtering and denoising.

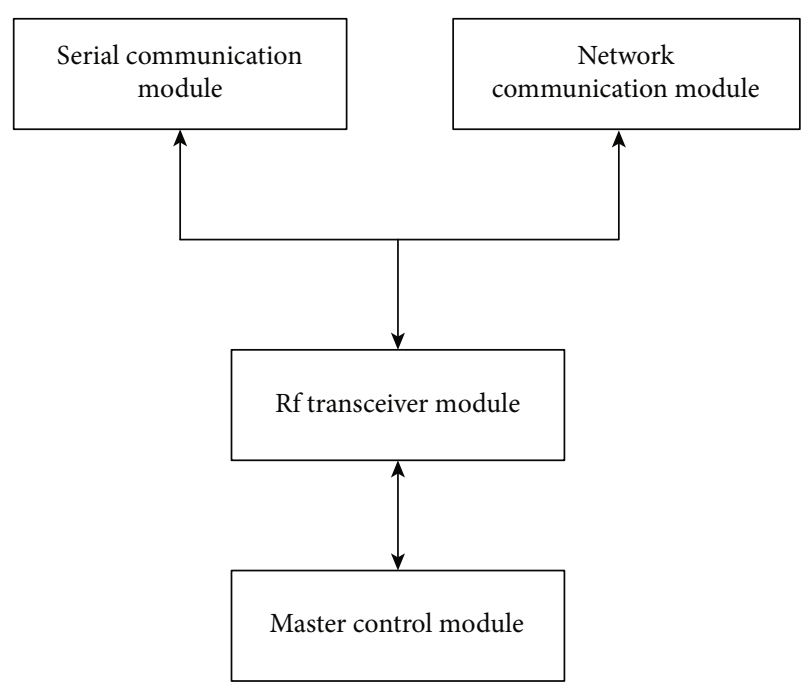

FIgURE 1: Schematic diagram of the structure of shooting angle information collection system.

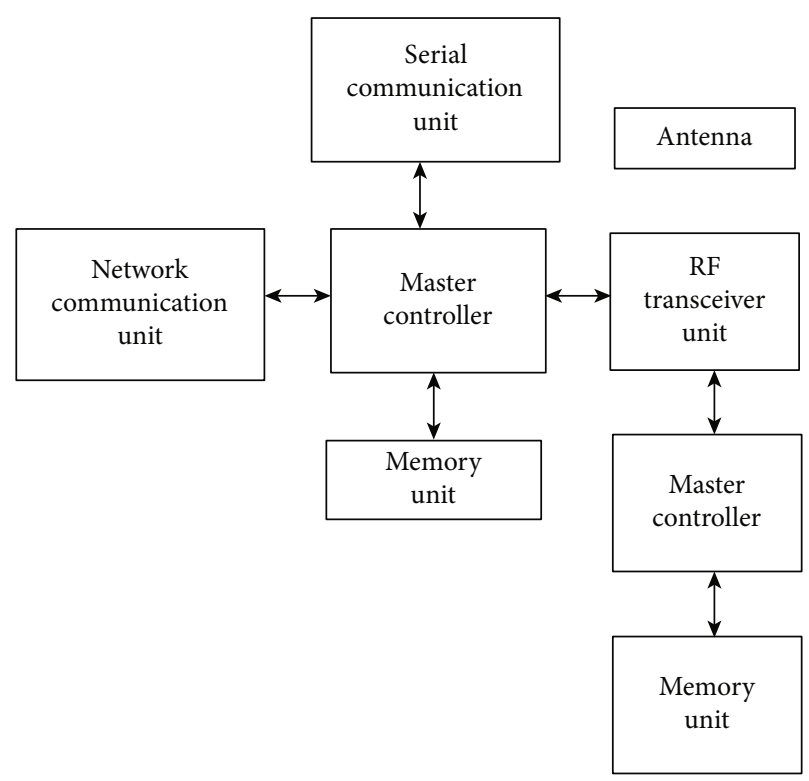

FIgURE 2: Volleyball information collection program.

2.2.1. Image Grayscale. Since the images acquired by the system are color images, the essence of image grayscale is to take the same values for the red component $R$, green component $G$, and blue component $B$. The processed color image realizes less postprocessing calculation and occupies less memory space. Grayscale image is an image with only one sampling color on any pixel. It is usually displayed as grayscale from the brightest black to white.

The component value interval of $R, G$, and $B$ is generally $[0,255]$, the grayscale is 256 , and grayscale images can represent 256 colors. The grayscale values are represented by Gray. In this paper, the weighted average method is used to realize the grayscale of color images.

Combining the three values $R, G$, and $B$, their respective weights are defined and a weighted average of them is calculated as follows: 


$$
R=G=B=\text { Gray }=\frac{\alpha R+\beta G+\gamma B}{3} .
$$

In equation (1), $\alpha, \beta$, and $\gamma$ represent the weights of $R, G$, and $B$, respectively. Since green produces the strongest stimulus effect on human eyes, followed by red and blue, grayscale images are obtained with higher quality when $\beta>\alpha>\gamma$. Combined with experience, it is known that the best grayscale images are obtained when $\alpha=0.299, \beta=0.587$, and $\gamma=$ $0.114[7,8]$. The expression for grayscale of color images is as follows:

$$
f(i, j)=0.299 R(i, j)+0.587 G(i, j)+0.114 B(i, j) .
$$

In equation $(2),(i, j)$ represents the point within the image, $f(i, j)$ represents the grayscale value after grayscale transformation, and the three values of this point in the initial color graphics $R, G$, and $B$ are described as $R(i, j), G(i, j)$, and $B(i, j)$, respectively.

2.2.2. Image Binarization. The essence of grayscale image binarization is to separate the foreground and background of the image [9]. Firstly, a threshold $T$ is set to divide the image into two parts, and the part above $T$ is called the foreground image and the part less than $T$ the background image. Assuming that the input and output images are represented as $f(x, y)$ and $g(x, y)$, respectively, the following equation is applied:

$$
g(x, y)= \begin{cases}1, & f(x, y) \geq T, \\ 0, & f(x, y)<T .\end{cases}
$$

If the target grayscale values are distributed in several nonadjacent regions, the following equation is applied:

$$
g(x, y)= \begin{cases}1, & f(x, y) \in \Phi, \\ 0, & \text { other. }\end{cases}
$$

In equation (4), $\Phi$ represents the set of different grayscale value intervals of the foreground target.

2.2.3. Filtering and Denoising Processing. In the process, the median filter algorithm is used to remove image noise. The median filter algorithm mainly applies the sorting statistics theory. The core of this algorithm is the sorting statistical theory, which belongs to a nonlinear smooth filtering image processing technique [10]. The filtering expression in the two-dimensional filtering window $W$ is as follows:

$$
g_{\text {median }}(x, y)=[f(s, t)] .
$$

In equation (5), $f(s, t)$ represents the grayscale value of the pixel in the neighborhood.

Through the above-mentioned implementation of image graying, binarization, filtering, and denoising processing operations on volleyball moving images, not only can the foreground information and background information in the picture be effectively separated but also the noise information in the image can be effectively removed, thereby improving the image information. Furthermore, the noise ratio lays the foundation for the subsequent volleyball shot feature extraction process.

\section{Automatic Recognition and Correction of Shot Angle Based on Feature Statistics}

3.1. Feature Extraction. Upon completion of image preprocessing, direct detection of feature points enables to avoid image segmentation, which is easier to implement. For this reason, this study implements feature point detection using approximate Hessian matrix based on Speeded-Up Robust Features (SURF). Assume that the Hessian matrix of the point $I\left(x^{\prime}, y^{\prime}\right)$ in the scale space that is $\sigma$ is represented as

$$
H=\left[\begin{array}{ll}
L_{x^{\prime} x^{\prime}}\left(x^{\prime}, \sigma\right) & L_{x^{\prime} y^{\prime}}\left(x^{\prime}, \sigma\right) \\
L_{x^{\prime} y^{\prime}}\left(x^{\prime}, \sigma\right) & L_{y^{\prime} y^{\prime}}\left(x^{\prime}, \sigma\right)
\end{array}\right]
$$

In equation (6), $L_{x^{\prime} x^{\prime}}\left(x^{\prime}, \sigma\right), L_{x^{\prime} y^{\prime}}\left(x^{\prime}, \sigma\right)$, and $L_{y^{\prime} y^{\prime}}\left(x^{\prime}, \sigma\right)$ are the results obtained from the second-order partial derivatives of the Gaussian function at $I\left(x^{\prime}, y^{\prime}\right)[11]$ and the convolution of the image $I\left(x^{\prime}, y^{\prime}\right)$.

On this basis, the image pyramid is constructed. In this process, the image needs to be resampled and then convoluted with Gaussian functions of different scales. A surf operator approximates the second-order Gaussian function through a box filter to improve the calculation speed. The image pyramids of different sizes can be generated in the image depending only on the filter size, and the convolution acceleration of the image is achieved using integral images $[12,13]$, and thus, the determinant of Hessian matrix is obtained as follows:

$$
\Delta H=D_{x^{\prime} x^{\prime}} D_{x^{\prime} y^{\prime}}\left(x^{\prime}\right)-\left(0.9 D_{y^{\prime} y^{\prime}}(x)\right)^{2}
$$

In equation (7), $\Delta$ indicates the response value of the box filter in the region near the point $I\left(x^{\prime}, y^{\prime}\right)$ [14], and $D_{x^{\prime} x^{\prime}}$, $D_{x^{\prime} y^{\prime}}$, and $D_{y^{\prime} y^{\prime}}$ represents the prefiltering image line, the filter convolution line, and the postfiltering image line, respectively. The value of the matrix determinant is used to judge the feature point, and if the value is positive and the two feature values are different, the point is defined as an extreme point.

Based on this, some among the extreme points are selected as candidate feature points. The selection method is to do the nonmaximum suppression in the $3 \times 3 \times 3$ regions centered on the extreme points and compare the adjacent scales with the 26 neighborhood values near the scale, and only the points that are larger or smaller than the neighborhood values can be identified as feature points.

To ensure the rotational invariance of the descriptors and give the reference direction for feature point assignment, a neighborhood with the feature point as the center of the circle and $6 s(s$ refers to the scale value where the feature point is located) as the radius is established firstly, and the Harr wavelet responses in different directions are computed in this 
region, while weights are attached to these response values to ensure that the weights of the adjacent feature points are larger; its rotation is performed in a circle by a sector template with a circular angle of sixty degrees to obtain the vector consisting of the cumulative sums of the Harr responses in all sliding windows, and the direction corresponding to the highest cumulative sum is set to be the reference direction of this feature point [15].

After determining the reference direction, construct a square with a side length of $20 \mathrm{~cm}$ along the direction centered on the feature point, and divide the window neighborhood into $4 \times 4$ subareas. In any subregion, the Harr wavelet response values of the sampling points are calculated for the reference direction $x^{\prime}$ and $y^{\prime}$, which are recorded as $d_{x^{\prime}}$ and $d_{y^{\prime}}$ and weighted, and the statistical results of different subregion response values are expressed as $\Sigma_{d^{x^{\prime}}}, \Sigma_{d^{y^{\prime}}},\left|\Sigma_{d^{x^{\prime}}}\right|,\left|\Sigma_{d^{y^{\prime}}}\right|$; then, a four-dimensional vector $V=\left[\Sigma_{d^{x^{\prime}}}, \Sigma_{d^{y^{\prime}}},\left|\Sigma_{d^{x^{\prime}}}\right|,\left|\Sigma_{d^{y^{\prime}}}\right|\right]$ is obtained, which is the feature descriptor of this subregion.

\subsection{Feature Statistical Classification Based on Support Vector} Machine Classifier. The feature extraction of volleyball players' shot angle has been completed above, and the next step is to classify and recognize the extracted features.

The greatest advantage of SVM is its excellent learning and generalization ability [16]. The main idea of SVM is to construct the plane by mapping the initial nonlinear data into a high-dimensional space and to maximize the distance from all sample sets to this plane [17]. For this purpose, this study uses the linear support vector machine in a linearly separable case to obtain the optimal classification hyperplane.

Assume that the training set is represented as

$$
T^{\prime}=\left\{\left(x_{1}^{*}, y_{1}^{*}\right),\left(x_{2}^{*}, y_{2}^{*}\right), \cdots,\left(x_{i}^{*}, y_{i}^{*}\right), \cdots,\left(x_{N}^{*}, y_{N}^{*}\right)\right\}
$$

In equation (8), the sample type $\left(x_{i}^{*}, y_{i}^{*}\right) \in\{0,1\}$, and $N$ represents the number of samples. Then, linear separability means that a certain classification plane is capable of correctly classifying all the data in the set, and the hyperplane is expressed as

$$
\omega \cdot x^{*}+b=0
$$

In equation (9), $\omega$ represents the Euclidean space dimension, $x^{*}$ represents the data set, and $b$ represents the flat subset. The classification decision function relative to the hyperplane is as follows [18]:

$$
f(x)=\operatorname{sign}\left(\omega \cdot x^{*}+b\right) .
$$

The distance margin between two planes is referred to as the classification interval between two sets of classified samples [19], and a larger value indicates a higher classification accuracy. Its expression is as follows:

$$
\operatorname{margin}=\frac{2}{|\omega|}
$$

In the case that all the two types of classified samples are correct, the classification interval is maximized when $\omega$ has the minimum value. Then, the optimal classification of linear separability can be expressed by the following equation:

$$
\left\{\begin{array}{l}
\min _{\omega, b} \frac{1}{2}|\omega|^{2}, \\
y_{i}\left(\omega \cdot x_{i}^{*}+b\right)-1 \geq 0 .
\end{array}\right.
$$

Using the Lagrangian function to calculate equation (12), assume that the function is expressed as

$$
L^{\prime}(\omega, b, a)=\frac{1}{2} \omega^{T} \omega-\sum_{i=1}^{l} a_{i}\left\{y_{i}\left[\omega \cdot x_{i}^{\prime}+b\right]-1\right\} \text {. }
$$

In equation (13), $a_{i}(i=1,2, \cdots, l)$ represents the Lagrangian operator.

Then, the first-order partial differentiation is performed on $b$ and $\omega$ so that it is equal to 0 :

$$
\left\{\begin{array}{l}
\partial L^{\prime}=\sum_{i=1}^{l} y_{i}^{*} a_{i}=0, \\
\partial b \\
\partial L^{\prime}=\omega-\sum_{i=1}^{l} y_{i}^{*} a_{i} x_{i}^{*}=0 . \\
\partial \omega
\end{array}\right.
$$

Equation (14) is substituted into equation (13) to obtain the dual optimization problem as follows:

$$
\max _{\alpha}-\frac{1}{2} \sum_{i=1}^{N} \sum_{j=1}^{N} a_{i} a_{j} x_{i}^{*} y_{j}^{*}\left(x_{i}^{*} \cdot x_{j}^{*}\right)-\sum_{i=1}^{N} a_{i} .
$$

Then, the optimal classification function can be expressed as follows:

$$
\begin{gathered}
f(x)=\min \frac{1}{2} \sum_{i=1}^{N} \sum_{j=1}^{N} a_{i} a_{j} x_{i}^{*} y_{j}^{*}\left(x_{i}^{*} \cdot x_{j}^{*}\right)-\sum_{i=1}^{N} a_{i}, \\
s \cdot t \cdot \sum_{i=1}^{N} x_{i}^{*} y_{i}^{*}=0, \quad a_{i} \geq 0, i=1,2, \cdots, N .
\end{gathered}
$$

In summary, the statistical classification of players' shot angle features can be achieved by using the optimal classification function.

3.3. Feature Recognition and Correction. In the process of lens angle recognition, this research realizes the final recognition combined with data features. For each acquired image, a $q_{\text {word }}$-dimensional statistical histogram is obtained after the description of the key frame feature library, and the shot angle recognition is performed by using the histogram intersection kernel support vector machine. The expression of the histogram intersection kernel is as follows:

$$
K_{h i}(u, v)=\sum_{i=1}^{m}\left\{u_{i}, v_{i}\right\}
$$




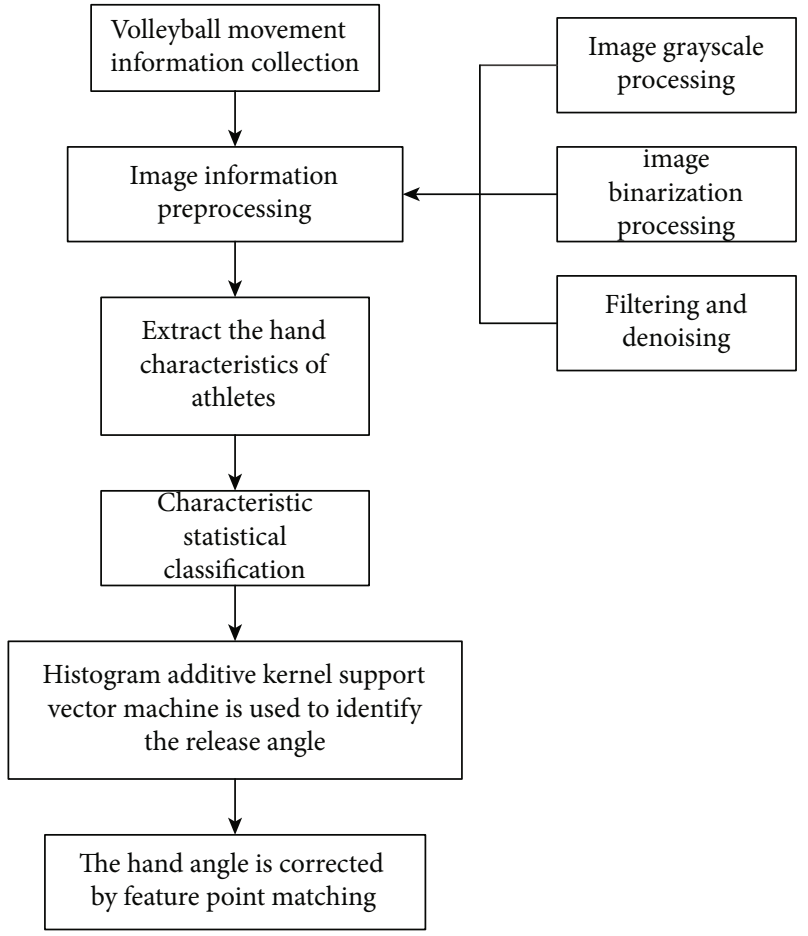

FIGURE 3: The realization process of the automatic recognition and correction method of volleyball players' shooting angle based on feature statistics.

In equation (17), $u=\left(u_{1}, u_{2}, \cdots, u_{i}, \cdots, u_{m}\right)$ and $v=\left(v_{1}\right.$, $\left.v_{2}, \cdots, v_{i}, \cdots, v_{m}\right)$ represent the statistical histograms of $m$ species, respectively.

The image features are then described to obtain a statistical histogram, and with intersection kernel SVM, a linear combination is made between the probabilities of occurrence of different angles, and the highest probability value after the combination is taken as the recognition result [20], and the algorithmic procedure is shown in

$$
\begin{gathered}
P^{(o)}=p_{1}^{(o)}, p_{2}^{(o)}, \cdots, p_{\text {action }}^{(o)}, \\
M=\operatorname{index}\left(\max \left(\sum_{o=1}^{2} \partial_{o} P^{(o)}\right)\right) .
\end{gathered}
$$

In the above equations, action represents the number of different shot angles, $o$ represents the extracted species features, $P^{(o)}$ represents the probability combination of occurrence of tested images, and $\partial_{o}$ represents the decision coefficient.

Feature point matching is performed for the recognition results and the feature library images in the acquisition system to derive the difference between the players' shot angle and the standard angle, so that the wrong shot angle can be corrected more accurately. For this purpose, this paper uses the paradigm of the ratio of nearest neighbor and second nearest neighbor for bidirectional matching. The equation is as follows:
TABLe 1: Simulation environment settings.

\begin{tabular}{lc}
\hline Name & Configuration \\
\hline Operating system & Windows 10, 32位 \\
CPU & Intel(R) Core(TM) i5-3230M@2.6GHZ \\
RAM & $8 \mathrm{~GB}$ \\
Development tools & Visual Studio 2019, OpenCV 2.3.8 \\
Optical camera model & L5163MX140-4 \\
\hline
\end{tabular}

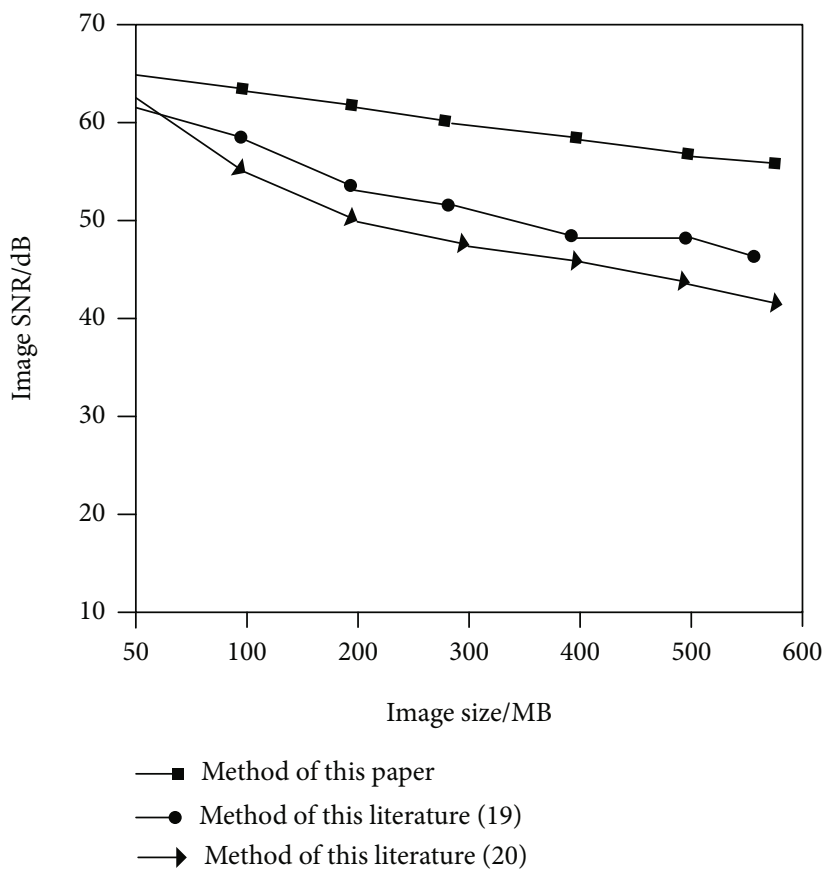

FIgURE 4: Comparison of image signal-to-noise ratio of different methods.

$$
D^{\prime}=\sqrt{\left(\varsigma_{1}-\varsigma_{1}^{\prime}\right)^{2}+\left(\varsigma_{2}-\varsigma_{2}^{\prime}\right)^{2}+\cdots\left(\varsigma_{\eta}-\varsigma_{\eta}^{\prime}\right)^{2}} .
$$

In equation (20), $\left(\varsigma_{1}, \varsigma_{2}, \cdots, \varsigma_{\eta}\right)$ and $\left(\varsigma_{1}^{\prime}, \varsigma_{2}^{\prime}, \cdots, \varsigma_{\eta}^{\prime}\right)$ represent the eigenvectors of the two feature points.

In summary, the design of the automatic recognition and correction method of volleyball players' shooting angle based on feature statistics is completed, and the realization process is shown in Figure 3.

\section{Simulation Experimental Data Analysis and Research}

In order to verify the feasibility of the automatic recognition and correction method of volleyball players' shot angle based on feature statistics designed above, the following simulation experiment is set up. The simulation environment is shown in Table 1.

In the experiment, volleyball spike is taken as an example. Assume that the ball speed is $80 \mathrm{~km} / \mathrm{h}$ and the height of the hitting point is about $3.2 \mathrm{~m}$. Firstly, 10 images of players' spike 


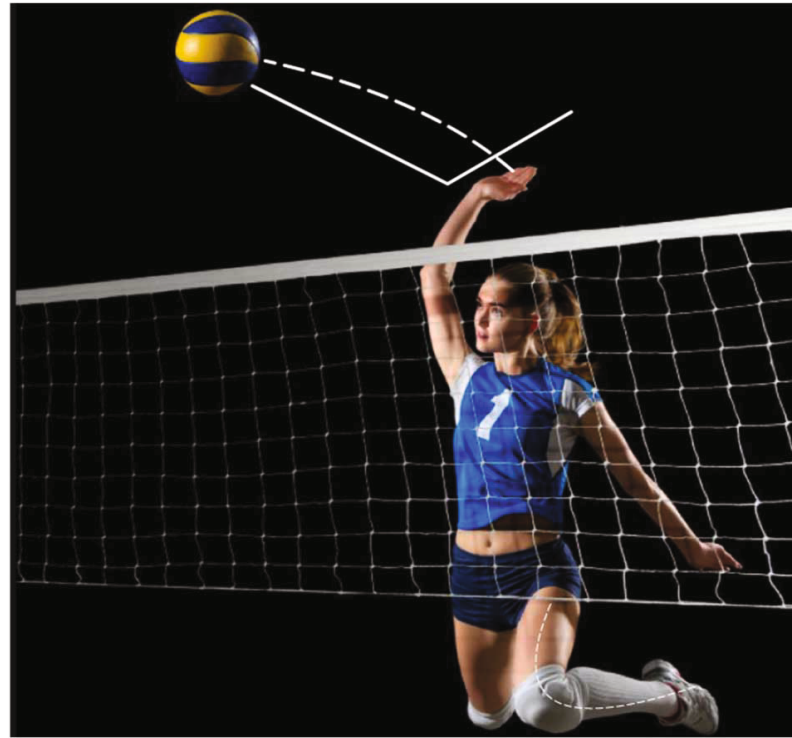

FIGURE 5: Elbow and hand shot angle.

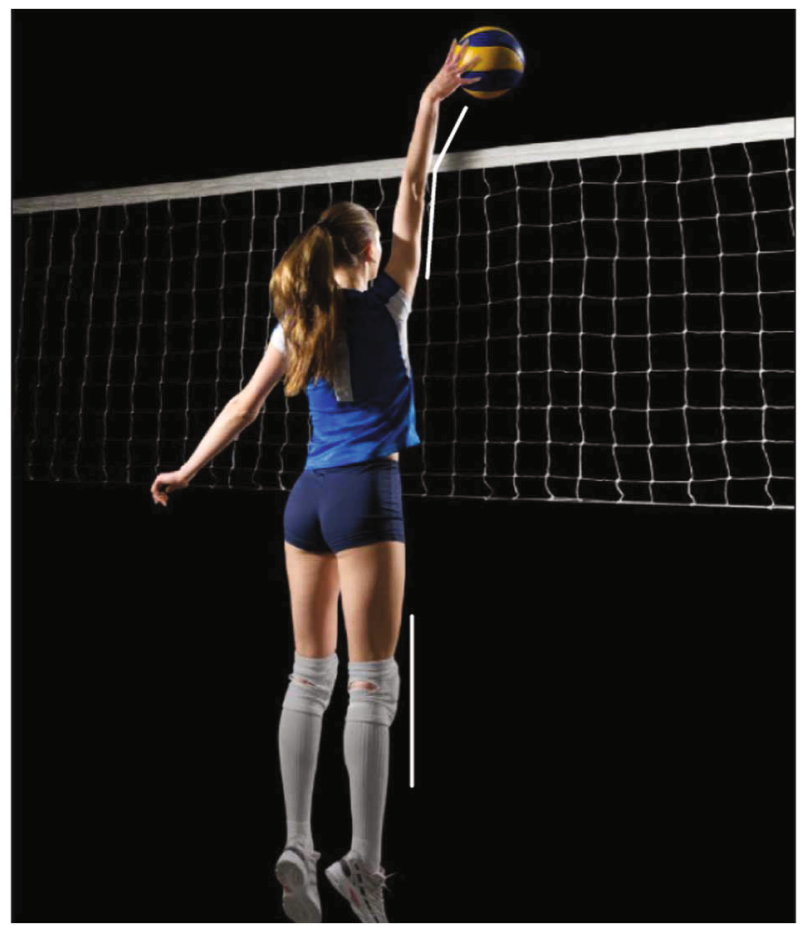

Figure 6: Leg angle change.

shot angles are acquired using Gabor filter and feature extraction is performed using an $8 \times 8$ grid. Before feature extraction, image denoising is performed using the method in this paper, 3D shear domain image denoising in the literature [19], and convolutional neural network-based denoising method in the literature [20]. The final image signal-to-noise ratio obtained is shown in Figure 4.

It can be seen from Figure 4 that with the increase of image size, the image signal-to-noise ratio decreases after denoising by the three methods. The decreasing trend of the image signal-to-noise ratio is not obvious after applying the method in this paper, and it always remains high. This indicates that the median filtering algorithm in this study can effectively denoise images, which lays a good foundation for the subsequent feature statistics.

After the image processing mentioned above, the shot angle is recognized using the recognition algorithm in this paper. A variety of features such as players' legs, elbows, and hands are counted in the experiment to more accurately recognize whether the shot angle is correct. The recognition results are shown in Figures 5 and 6.

As shown in Figures 5 and 6, through the statistics of multiple image frame features, this study recognizes that the angle between the arm and the right angle of the volleyball is greater than 90 degrees and the knee bending is closer to 90 degrees during the spike action in Figure 5. This kind of shot angle is conducive to release force, thus improving the spike effect, while in Figure 6, the player's arm and knee bending degree is smaller and the body weight falls on the feet, which affects the jump, thus reducing the spike strength.

\section{Conclusion}

In this study, a volleyball sports information acquisition system is designed to acquire images of the players' shot angles, and after a series of processing, the images are obtained with high signal-to-noise ratio, while feature extraction is completed based on the key features of each frame, and the feature statistics are accomplished through a support vector machine classifier to achieve the recognition of the shot angles, which helps coaches to correct nonstandard movements. The method features strong image processing capability and can accurately complete image recognition, which will play an important supporting role in sports training.

In the future research, a variety of positioning measurement technologies such as computer vision, machine vision, and ultrasonic positioning can be introduced, and the advantages of different methods can be used to obtain a more ideal image acquisition effect and make volleyball training more scientific.

\section{Data Availability}

The labeled dataset used to support the findings of this study are available from the corresponding author upon request.

\section{Conflicts of Interest}

The author declares no competing interests.

\section{References}

[1] C. A. DiCesare, A. Montalvo, K. D. B. Foss et al., "Sport specialization and coordination differences in multisport adolescent female basketball, soccer, and volleyball athletes," Journal of Athletic Training, vol. 54, no. 10, pp. 1105-1114, 2019.

[2] H. M. Yin, P. Sun, G. U. Song et al., "The specific physical ability basic feature of volleyball players during the game," Journal 
of Beijing University of Physical Education, vol. 9, pp. 15-23, 2019.

[3] P. Iodice, P. Ripari, and G. Pezzulo, "Local high-frequency vibration therapy following eccentric exercises reduces muscle soreness perception and posture alterations in elite athletes," European Journal of Applied Physiology, vol. 119, no. 2, pp. 539-549, 2019.

[4] H. Zhi, T. Ma, D. Pei et al., "Magnetic target recognition and localization method unaffected by attitude," EPL (Europhysics Letters), vol. 129, no. 4, pp. 49-55, 2020.

[5] X. Zheng, X. Chen, and X. Lu, "A joint relationship aware neural network for single-image 3D human pose estimation," IEEE Transactions on Image Processing, vol. 29, pp. 47474758, 2020.

[6] J. Nye, L. Zingaretti, and M. Perez-Enciso, "224 automatic image feature extraction for genetic analysis in cattle," Journal of Animal Science, vol. 97, Supplement_3, pp. 47-47, 2019.

[7] B. Kumar, O. Dikshit, A. Gupta, and M. K. Singh, "Feature extraction for hyperspectral image classification: a review," International Journal of Remote Sensing, vol. 41, no. 16, pp. 6248-6287, 2020 .

[8] K. Ichiyama, "Image processing apparatus, image pickup apparatus and image processing method," Journal of Oral Rehabilitation, vol. 8, no. 3, pp. 203-208, 2018.

[9] M. Rezaei Abkenar, H. Sadreazami, and M. O. Ahmad, "Salient region detection using feature extraction in the nonsubsampled contourlet domain," IET Image Processing, vol. 12, no. 12, pp. 2275-2282, 2018.

[10] T. Santos, S. Schrunner, B. C. Geiger et al., "Feature extraction from analog Wafermaps: a comparison of classical image processing and a deep generative model," IEEE Transactions on Semiconductor Manufacturing, vol. 32, no. 2, pp. 190-198, 2019.

[11] S. Echegaray, S. Bakr, D. L. Rubin, and S. Napel, "Quantitative Image Feature Engine (QIFE): an open-source, modular engine for 3D quantitative feature extraction from volumetric medical images," Journal of Digital Imaging, vol. 31, no. 4, pp. 403-414, 2018.

[12] R. B. Shang, R. Archibald, A. Gelb, and G. P. Luke, "Sparsitybased photoacoustic image reconstruction with a linear array transducer and direct measurement of the forward model (erratum)," Journal of Biomedical Optics, vol. 24, no. 8, pp. 1-9, 2019.

[13] Y. Zhang, S. S. Zhu, J. Lin, and P. Jin, "High-quality panchromatic image acquisition method for snapshot hyperspectral imaging Fourier transform spectrometer," Optics Express, vol. 27 , no. 20 , pp. 28915-28928, 2019.

[14] S. Bazeille, Y. Maillot, F. Cordier, C. Riou, and C. Cudel, "Light-field image acquisition from a conventional camera: design of a four minilens ring device," Optical Engineering, vol. 58, no. 1, pp. 1-9, 2019.

[15] S. Routray, A. K. Ray, C. Mishra, and G. Palai, "Efficient hybrid image denoising scheme based on SVM classification," Optik, vol. 157, pp. 503-511, 2018.

[16] S. Javed, A. Mahmood, S. al-Maadeed, T. Bouwmans, and S. K. Jung, "Moving object detection in complex scene using spatiotemporal structured-sparse RPCA," IEEE Transactions on Image Processing, vol. 28, no. 2, pp. 1007-1022, 2019.
[17] X. Liu, Y. Tang, and Y. Yang, "Primal-dual algorithm to solve the constrained second-order total generalized variational model for image denoising," Journal of Electronic Imaging, vol. 28 , no. 4, pp. 1-15, 2019.

[18] S. Seo, "Image denoising and refinement based on an iteratively reweighted least squares filter," KSCE Journal of Civil Engineering, vol. 24, no. 3, pp. 943-953, 2020.

[19] S. Zhang, L. Wang, C. Chang, C. Liu, L. Zhang, and H. Cui, "An image denoising method based on BM4D and GAN in 3D shearlet domain," Mathematical Problems in Engineering, vol. 2020, Article ID 1730321, 11 pages, 2020.

[20] C. Shan, X. Guo, and J. Ou, "Residual learning of deep convolutional neural networks for image denoising," Journal of Intelligent \& Fuzzy Systems, vol. 37, no. 2, pp. 2809-2818, 2019. 